\title{
cDNA cloning and characterization of two trehalases from Spodoptera litura (Lepidoptera; Noctuidade)
}

\author{
Q. Zou*, P. Wei*, Q. Xu, H.Z. Zheng, B. Tang and S.G. Wang \\ Hangzhou Key Laboratory of Animal Adaptation and Evolution, \\ Hangzhou Normal University, Hangzhou, Zhejiang, China \\ *These authors contributed equally to this study. \\ Corresponding author: S.G. Wang \\ E-mail: sgwang@mail.hz.zj.cn
}

Genet. Mol. Res. 12 (2): 901-915 (2013)

Received June 15, 2012

Accepted January 1, 2013

Published April 2, 2013

DOI http://dx.doi.org/10.4238/2013.April.2.7

\begin{abstract}
The oriental leafworm moth, Spodoptera litura, is a major agricultural pest in southeast Asia and nearby Pacific regions. Two distinct trehalases have been identified in insects: soluble trehalase (Treh1) and membrane-bound trehalase (Treh2), although there is currently no information on these genes in S. litura. To characterize the distribution and function of treh, cDNAs of Treh proteins were cloned from S. litura. SpoliTreh1 cDNA has an open reading frame of 1758 nucleotides, which encodes a protein of 585 amino acids, with a predicted mass of approximately $67.07 \mathrm{kDa}$ and an isoelectric point of 4.86. SpoliTreh2 cDNA has an open reading frame of 2325 nucleotides, encoding a protein of 645 amino acids, a mass of approximately 73.62 $\mathrm{kDa}$, and an isoelectric point of 5.90. Northern blotting analysis revealed that SpoliTreh1 transcripts are in the midgut, fat body, tracheae, and epidermis, but not in the brain and Malpighian tubules of $S$. litura larvae, whereas SpoliTreh2 transcripts were found in all 6 tissues. SpoliTreh1 transcripts were highly expressed in the fat body of the prepupal stage, and SpoliTreh2 transcripts were highly expressed in the fat body of 3-day-old larvae of the 6th instar and during the 1st 6 days of the pupal stage, except the 2nd day. Both SpoliTreh1 and SpoliTreh2
\end{abstract}


were highly expressed in third-instar larvae.

Key words: Trehalase; Cloning; Characterization; Expression pattern; Spodoptera litura

\section{INTRODUCTION}

Carbohydrates are a primary source of energy for the maintenance of metabolism in insects (Mariano et al., 2009), and trehalose is the main carbohydrate in insects. Trehalose is present in almost all forms of life except mammals (Lee et al., 2001; Elbein et al., 2003; Frison et al., 2007; Silva et al., 2010). It protects cellular membranes and proteins from oxidation, dehydration, heat, freezing, desiccation, and cold owing to its higher glass transition temperature compared with those to other sugars (Crowe et al., 1984; Eleutherio et al., 1993; Sun and Davidson, 1998; Davidson and Sun, 2001).

Trehalase (encoded by 2 genes, Treh1 and Treh2) is an anomer-inverting $\alpha$-trehalose1-D-glucosidase, which plays an important role in the use of carbohydrates by hydrolyzing trehalose molecules into 2 glucose molecules (Tang et al., 2008). To date, 2 forms of trehalase have been found and cloned in several insect species (Wyatt, 1967), including Apis mellifera (Lee et al., 2007), Bombyx mori (Mitsumasu et al., 2005), Omphisa fuscidentalis (Tatun et al., 2008), Pimpla hypochondriaca (Parkinson et al., 2003), Spodoptera exigua (Tang et al., 2008), and Tenebrio molitor (Takiguchi et al., 1992). Initial studies of trehalose and protein purification have found that the main distribution of soluble trehalase (Treh1) and membrane-bound trehalase (Treh2) sometimes differed (Tang et al., 2008), which might indicate different functions.

In the silkworm B. mori, Treh1 is predominantly expressed in the pre-pupal and pupal midgut, whereas Treh2 expression is almost constant in the midgut, although some fluctuations do occur (Yamashita et al., 1974; Sumida and Yamashita, 1977; Mitsumasu et al., 2008). Ujita et al. (2011) found that the oligosaccharide chains of insect trehalase are essential for soluble trehalase expression and enzymatic activity. Treh2 is mainly found in mitochondrial membranes as well as in brain, cuticle, and midgut and is believed to be a transmembrane enzyme with an active site on the outside of the cell membrane (Mitsumasu et al., 2008; Tang et al., 2008). Wegener et al. (2003) suggested that Treh2 comprises an overt form and a latent form that can be activated by destroying membrane integrity in flight muscle cells.

Treh1 and Treh2 use different sources of trehalose. Treh1 is located in intracellular hydrolyzing endogenous trehalose, and Treh2 is a transmembrane enzyme involved in the assimilation of exogenous trehalose to supply energy (de Almeida et al., 2009). Chen et al. (2010) found that the expression of trehalose is increased and that of glucose is reduced with Treh1 reduction, whereas Treh2 occurs in S. exigua through the RNA interference.

$S$. litura is an insect pest that belongs to the order Lepidoptera. It can devour crops in a surprisingly short time and migrates long distances. In this study, Treh1 and Treh2 in S. litura were cloned to evaluate their transcriptional regulation. The study determined that trehalase activity in S. litura tissues varied during every developmental period, which suggested that gene expression was diverse. Comparison and analysis characterized these genes and their tissue expression, revealing their relationship and the method through which they regulate the catabolic pathways of trehalose. In addition, this study may contribute to the development of new agents that inhibit trehalose by blocking catabolic pathways through which trehalose acts on trehalases. These agents could replace insecticides for the control of S. litura. 


\section{MATERIAL AND METHODS}

\section{Insect cultures}

S. litura have been maintained in our laboratory for more than 3 years. Larvae were reared on an artificial diet at $25^{\circ} \pm 1^{\circ} \mathrm{C}$ under a 14-h:10-h light-dark photoperiod. The developmental stages were synchronized at each molt by collecting new larvae or pupae. The brain, midgut, fat body, epidermis, and other tissues from first-instar larvae to pupae were dissected in saline solution $(0.75 \% \mathrm{NaCl})$ and stored at $-80^{\circ} \mathrm{C}$ for future analysis.

\section{RNA extraction, cDNA synthesis, and polymerase chain reaction (PCR)}

Total RNA was extracted from the fat body of $S$. litura pupae using the Trizol (Invitrogen) method. The fat body $(100 \mathrm{mg})$ was added into a tube containing liquid nitrogen and quickly ground. The homogenates were transferred to a new tube containing $1 \mathrm{~mL}$ Trizol, vortexed for $3 \mathrm{~min}$, and incubated at room temperature for $5 \mathrm{~min}$. Chloroform $(200 \mu \mathrm{L})$ was added, and the mixture was incubated at room temperature for an additional $5 \mathrm{~min}$. The mixture was centrifuged at $12,000 \mathrm{~g}$ for $15 \mathrm{~min}$ at $4^{\circ} \mathrm{C}$. The supernatant was transferred to a new tube, $500 \mu \mathrm{L}$ isopropanol was added, and the mixture was incubated at room temperature for $10 \mathrm{~min}$. After centrifugation at $12,000 \mathrm{~g}$ for $10 \mathrm{~min}$ at $4^{\circ} \mathrm{C}$, the supernatant was discarded, and the RNA pellet was washed with $75 \%$ ethanol, dissolved in diethylpyrocarbonate double-distilled water and stored at $-80^{\circ} \mathrm{C}$ until further use. A 1- $\mu \mathrm{g}$ sample of total RNA was reverse-transcribed at $42^{\circ} \mathrm{C}$ for $1 \mathrm{~h}$ in a $10-\mu \mathrm{L}$ reaction solution containing reaction buffer, $10 \mathrm{mM}$ dithiothreitol, $0.5 \mathrm{mM}$ deoxyribonucleotide triphosphates, $0.5 \mu \mathrm{g}$ oligo-d(T) 18 , and reverse transcriptase from avian myeloblastosis virus.

Four pairs of primers were designed for Treh1 and Treh2 (Treh1-DF1 and Treh1-DF2, Treh1-DR1 and Treh1-DR2, Treh2-DF1 and Treh2-DF2, and Treh2-DR1 and Treh2-DR2; Table 1) based on the conserved nucleotide sequences of known trehalase proteins. An initial PCR was performed with primers Treh1-DF1 (or Treh2-DF1) and Treh1-DR1 (or Treh2-DR1) according to the following conditions: 3 cycles of $30 \mathrm{~s}$ at $94^{\circ} \mathrm{C}, 30 \mathrm{~s}$ at $45^{\circ} \mathrm{C}$, and $120 \mathrm{~s}$ at $72^{\circ} \mathrm{C}$, followed by 28 cycles of $30 \mathrm{~s}$ at $94^{\circ} \mathrm{C}, 30 \mathrm{~s}$ at $48^{\circ} \mathrm{C}$, and $70 \mathrm{~s}$ at $72^{\circ} \mathrm{C}$. A second PCR was carried out using the nested primers Treh1-DF2 (or Treh2-DF2) and Treh1-DR2 (or Treh2DR2) under the same conditions as those described for the initial PCR (Yamoah et al., 2008). The products were subjected to agarose gel electrophoresis. The true electrophoretic DNA bands corresponding to the expected size of approximately 800-1000 bp were excised from the agarose gel and purified using a DNA gel extraction kit. These PCR products were cloned into a pMD18-T vector and sequenced using the dideoxynucleotide method.

\section{Rapid amplification of cDNA ends (RACE)}

Specific primers for 5'-RACE (SpoliTreh1-5RA/SpoliTreh2-5RA and SpoliTreh1-5RB/ SpoliTreh2-5RB) and 3'-RACE(SpoliTreh1-3FA/SpoliTreh2-3FA and SpoliTreh1-3FB/SpoliTreh23FB; see Table 1) were synthesized based on the cDNA sequence of the PCR fragment. 5'-RACE was performed using $2.5 \mu \mathrm{L}$ 5'-ready cDNA with Universal Primer Mix and SpoliTreh1-5RA/ SpoliTreh2-5RA. Nested PCR was then carried out with Nested Universal Primer A and SpoliTreh15RB/SpoliTreh2-5RB. 3'-RACE was performed using $2.5 \mu \mathrm{L}$ 3'-ready cDNA with Universal 
Table 1. Cloning strategy for Spolitreh cDNA and probe primers for Northern blotting.

\begin{tabular}{|c|c|c|c|c|}
\hline \multirow[t]{2}{*}{ PCR fragment } & \multicolumn{4}{|c|}{ Primers } \\
\hline & Name & Direction & Type & Nucleotide sequence $\left(5^{\prime}-3^{\prime}\right)$ \\
\hline \multirow[t]{11}{*}{1} & Treh1-DF1 & $\mathrm{F}$ & $\mathrm{D}$ & GGA RWT YTA YTA CTG GGA \\
\hline & Treh1-DF2 & $\mathrm{F}$ & $\mathrm{D}$ & TGG GAT HRT BRA AGG KCT \\
\hline & Treh1-DF3 & $\mathrm{F}$ & $\mathrm{D}$ & GAG AGY GGY TGG GAY TTC TC \\
\hline & Treh1-DR1 & $\mathrm{R}$ & $\mathrm{D}$ & CCR TTB SWC CAY CCG AA \\
\hline & Treh1-DR2 & $\mathrm{R}$ & $\mathrm{D}$ & CGC RTC RTA YTT CTC RAA CAT \\
\hline & Treh1-DR3 & $\mathrm{R}$ & $\mathrm{D}$ & GCC ADG CGT TRG GGW AGT CC \\
\hline & Treh2-DF1 & $\mathrm{F}$ & $\mathrm{D}$ & TAC TGG GAY TCB TAC TGG A \\
\hline & Treh2-DF2 & $\mathrm{F}$ & $\mathrm{D}$ & ACG GNG GMM GVA THT ACT A \\
\hline & Treh2-DF3 & $\mathrm{F}$ & $\mathrm{D}$ & TCC CVG TNG ACY TGA ACG C \\
\hline & Treh2-DR1 & $\mathrm{R}$ & $\mathrm{D}$ & GCG TCG TAC TTY TCR ARC AT \\
\hline & Treh2-DR2 & $\mathrm{R}$ & $\mathrm{D}$ & GCC AVG CRT TSG GRT AGT CC \\
\hline 2 & Treh2-DR3 & $\mathrm{R}$ & $\mathrm{D}$ & CCA SRC NCC BAC NTC BTC GTG C \\
\hline \multirow{5}{*}{ 3'-RACE } & SpoliTreh1-3FA & $\mathrm{F}$ & G & GAG TGG AGA GCA ATG GGA C \\
\hline & SpoliTreh1-3FB & $\mathrm{F}$ & G & GAG TAT TGT AGT AAA CGC CA \\
\hline & SpoliTreh1-3FC & $\mathrm{F}$ & $\mathrm{G}$ & GGA ACA CCT GAG GCG AGT G \\
\hline & SpoliTreh2-3FA & $\mathrm{F}$ & G & GTC GGG ATT ACT TCT GCC \\
\hline & SpoliTreh2-3FB & $\mathrm{F}$ & G & GGA TTA CTA CGT GAA CAG AG \\
\hline 3 & SpoliTreh2-3FC & $\mathrm{F}$ & G & GAC ATA TTC GAC GGA GGC A \\
\hline \multirow[t]{5}{*}{ 5'-RACE } & SpoliTreh1-5RA & $\mathrm{R}$ & G & GTG GCT ACT ACC TTC TTT CTC \\
\hline & SpoliTreh1-5RB & $\mathrm{R}$ & G & CCA GCC AGA AGT CCA TTT CC \\
\hline & SpoliTreh1-5RC & $\mathrm{R}$ & G & GTG TCG TTG GTG TAT TGG \\
\hline & SpoliTreh2-5RA & $\mathrm{R}$ & G & GGT CCT TGT GAT TGG TCG \\
\hline & SpoliTreh2-5RB & $\mathrm{R}$ & G & GAC TTC AAT GGT GTG GTT GG \\
\hline 4 & SpoliTreh2-5RC & $\mathrm{R}$ & G & TGG ATG TGT TGT CTG AGG \\
\hline \multirow[t]{6}{*}{ Probe } & SpoliTreh1-PFA & $\mathrm{F}$ & G & CTC TGA TGA AGG CGA ACT CG \\
\hline & SpoliTreh1-PFB & $\mathrm{R}$ & G & CAT TTG GCC TCT TCT TGC CCG \\
\hline & SpoliTreh1-PR & $\mathrm{F}$ & G & CCA ACC ACT CTC AGC TGC AC \\
\hline & SpoliTreh2-PFA & $\mathrm{R}$ & G & CAG TTG GGC AGG AAG ATG AAG C \\
\hline & SpoliTreh2-PFB & $\mathrm{F}$ & G & CTG TTA TTG TAC CTG GTG G \\
\hline & SpoliTreh2-PR & $\mathrm{R}$ & G & CAC CTG TTC AAT GGC ATC C \\
\hline
\end{tabular}

The full-length Treh cDNA sequences were determined by sequencing two overlapping PCR fragments 1 using fat body cDNA as a template. PCR fragments 2 and 3 were obtained from 3'- and 5'-RACE, respectively. PCR fragment 4 was used as a probe for Northern blot. $F=$ forward; $R=$ reverse; $D=$ degenerate primer; $G=$ genespecific primer; $A=$ nested universal primer O: 3'-RACE CDS Primer.

Primer Mix and SpoliTreh1-3FA/SpoliTreh2-3FA, and then with Nested Universal Primer and SpoliTreh1-3FB/SpoliTreh2-3FB. The PCR conditions were $10 \mathrm{~min}$ at $94^{\circ} \mathrm{C}$, followed by 30 cycles of $30 \mathrm{~s}$ at $94^{\circ} \mathrm{C}, 30 \mathrm{~s}$ at $60^{\circ} \mathrm{C}$, and $90 \mathrm{~s}$ at $72^{\circ} \mathrm{C}$, and then $10 \mathrm{~min}$ at $72^{\circ} \mathrm{C}$ (Tang et al., 2008).

The PCR products were subjected to electrophoresis. Two strong DNA bands corresponding to the expected size of approximately $1000 \mathrm{bp}$ were detected with SpoliTreh1 5'-RACE. Only 1 strong DNA band at approximately 700 bp was detected with SpoliTreh1 3'-RACE. For SpoliTreh2 5'- and 3'-RACE, 2 strong DNA bands corresponding to the expected size of approximately 830 and 990 bp were observed, respectively. All 5 bands were excised from the agarose gel and purified using a DNA gel extraction kit, cloned into a pMD18-T vector, and sequenced using the dideoxynucleotide method.

\section{Analysis of SpoliTreh cDNA sequences}

SpoliTreh1 and SpoliTreh2 cDNA sequences were compared with other trehalase sequences deposited in GenBank using Basic Local Area Search Tool-N and Basic Local Area Search Tool-X via the National Center for Biotechnology Information website. SpoliTreh1 and SpoliTreh2 amino acid sequences were deduced from the corresponding cDNA sequences using the translation tool on the ExPASy Proteomics website [http://expasy.org/tools/dna.html (accessed 
August 5, 2012)]. The neighbor-joining method was used to construct a phylogenetic tree with the Molecular Evolutionary Genetics Analysis 3.1 software based on the amino acid sequences of trehalase. A bootstrap analysis was carried out, and the robustness of each cluster was verified using 1000 replicates. Other protein sequence analysis tools used in this study were obtained from the ExPASy Proteomics website [http://expasy.org/ (accessed August 5, 2012)]. Multiple sequence alignment of insect Treh was performed using a tool at the MultAlin multiple sequence alignment website [http://bioinfo.genotoul.fr/multalin/multalin.html (accessed August 5, 2012)].

\section{Northern blot analysis of Treh tissue distribution}

Samples of $20 \mu \mathrm{g}$ total RNA were extracted from fat body, brain, midgut, epidermis, tracheae, and spermary of fifth-instar larvae and separated on a formaldehyde agarose gel containing ethidium bromide. The RNA was subsequently blotted onto a Hybond- $\mathrm{N}^{+}$membrane. Two Treh cDNA fragments of approximately 562 (SpoliTreh1) and 695 bp (SpoliTreh2) were labeled with digoxin and used as probes (Figure 1). Template DNA (1-3 $\mu \mathrm{g})$ was added to an Eppendorf tube and diluted with double-distilled water to a final volume of $16 \mu \mathrm{L}$. After the DNA was denatured through heating in a $100^{\circ} \mathrm{C}$ water bath for $10 \mathrm{~min}$, the solution was quickly cooled in an ice bath. At the same time, $4 \mu \mathrm{L}$ thoroughly mixed Dig-High Primer was added to the denatured DNA, mixed, and centrifuged to collect remnants clinging to the tube wall and force the flow to the bottom of tube. The mixture was incubated at $37^{\circ} \mathrm{C}$ in a water bath for $20 \mathrm{~h}$ and then heated to $65^{\circ} \mathrm{C}$ in a water bath for $10 \mathrm{~min}$ to stop the reaction. After hybridization, the membrane was washed with $0.2 \mathrm{X}$ SSPE $\left(0.03 \mathrm{M} \mathrm{NaCl}, 0.002 \mathrm{M} \mathrm{NaH}_{2} \mathrm{PO}_{4}, 0.0002 \mathrm{M}\right.$ ethylenediaminetetraacetic acid, $\mathrm{pH}$ 7.4) at $45^{\circ} \mathrm{C}$ and exposed to $\mathrm{X}$-ray film at $-70^{\circ} \mathrm{C}$ for $24 \mathrm{~h}$ (Tang et al., 2008; Tatun et al., 2008). Ribosomal RNA was used as a control under the same conditions.

\section{Developmental expression of Spolitrehs}

Samples of total RNA $(20 \mu \mathrm{g})$ isolated from 3 to 5 fat bodies of viable larva from day 1 of the 6 th instar to day 6 of the pupal stage and whole body from the 1st instar to 5 th instar were separated on a formaldehyde agarose gel containing ethidium bromide. The RNA was subsequently blotted onto a Hybond- $\mathrm{N}^{+}$membrane. Northern blotting was performed as described above.

\section{RESULTS}

\section{Sequence analysis of SpoliTreh cDNAs}

Two SpoliTreh cDNAs were obtained with 5'- and 3'-RACE. SpoliTreh1 cDNA (GU211891) had an open reading frame of 1758 nucleotides, which encoded a protein of 585 amino acids with a predicted molecular mass of approximately $67.07 \mathrm{kDa}$ and an isoelectric point of 4.86 (Figure 1A). SpoliTreh2 cDNA (GU211890) had an open reading frame of 2325 nucleotides, which encoded a protein of 645 amino acids with a predicted molecular mass of approximately $73.62 \mathrm{kDa}$ and an isoelectric point of 5.90 (Figure 1B). The 2 types of Treh had the same conserved amino acid sequence (GGGGEY). SpoliTreh showed a 36-96\% identity to other known Treh family genes, and Treh1 could be clearly distinguished from Treh 2 between different insects (Figure 2; Tables 2 and 3). SpoliTreh1 showed the highest identity (92\%) 
A

1 AGTCGCGTTCGTGTTTGCGACTCGAGGGCATGTAATCGATTAACTTATCCCAACGACATGTCAAATCGATAAAATCAATCGATTGTAAAGAAATGATATGATAATTTAATTTTGTTTAT 121 CTGTTACCAGTGTAAATCCTAGTGTAGTTGCGATTAGTTACGTGTATCGATATTTCGATTTTAAATTCAACTTGTGTTCGGTTGCCGGTGAGGTGAATGGTGTCCTTTTGTAAGATGCGG 241 GGataCCTGATTGTCCTGGTGGCGGCCGTTGCGCTGGCCAGTGCTGCTGACCTACGTCCCAGTTGCAGCAAGCCCGTCTACTGCGAAGGGGACTCCTCCATCGAGTCCAAATGGCCAGG

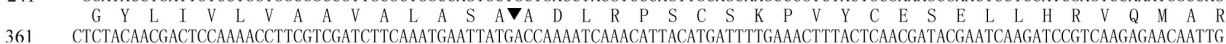
361- CICACAAGGACCCAAACCCG $R$ E F V N K Y F S D E G E L E E W T P P D F N S D P K F V Y T I K D K A L R E F A K N I N N I W P L L A R K V K K D K K V I O N P D R Y S L L V P I T H G F I I P G G - CGaticaCgGagad

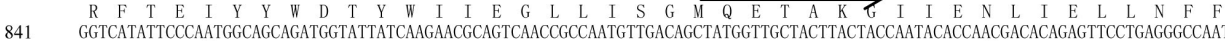

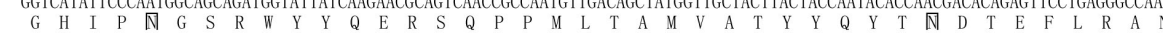
961 ATTGCATCTTTAGAGAAGGAAATGGACTTCTGGG GGATGAAAGACAGTGACCGTTGAGAAAGAAGGTAGTAGCCACAAACTGCTCAGATATTTCGCTCTCAGCTCGGGTCCAAGACC 1081 I I A S S L E E K E E M D F F W 1081 GAATCGTATTATGAAGATTATGAAAACGCAGTAGAATTCAGTGAACAAGGTCGCACAGACTTCTATATTGATATTAGGAGTGCAGCTGAGAGTGGTTGGGACTTCTCAACGCGGTGGTTC E S S Y Y Y GTCAACAATGATGGCAGCAACAACGGAACCTTAAAAGACATTCACACCAGATACGTCATACCTGTGGATCTAAATGCCATATTTGCCGGTGCTCTCCAAAACGTGGCAAACTTTAACGCO ATTTTGATGAACCCTCGCAAGGCTGGCACGTATGGCCAATTGGCTCAACAGTGGaGAGATGCCATTCAGTCGATTTGGTGGAATGAAGAAGAGGGAATGTGGTATGATTATGACATCAGA I L M N P R K A A T Y G Q L A Q Q Q W R D D A I Q Q S I L W N E E E G M W Y D Y Y D I R GACAAGTTACATCGCAAATATTTTTACTCATCTAACGTCTCTCCGTTGTGGCAACATGCTGTTGATCCGAATATAGTGAAAGCAAATELAGACAGAATTCTGAATAATCTTAAACAATCT

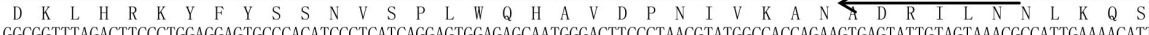

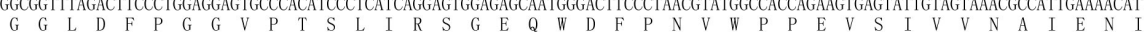
GGAC $G$ T P E A S V L A F F E T A Q T F V R S C H W W G F L E Y K Q M F E K K Y D A E N P G AAGTTCGGAGGAGGTGGGGATATAACGTGCAATTTGGTTTCGGATGGAGCAACGGTGCAGTGTTAGAGTTTATGAAGAAATATGGAGAAGGTTTGACTGCAGACGACTCTAACGACTTA $\underset{\text { GCTACTACTGCATCTCCCTCCAACAATAGGGATACTTCGAATAATACCGCATGACTAGGCGTGGGTGTAGTAAATAACTTTTTAATCTTTCTATCAGCATTCAAAATATTTTTTGATATT }}{\mathrm{K} \underset{\mathrm{F}}{\mathrm{G}} \mathrm{G}}$

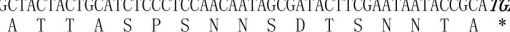

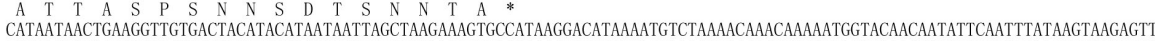
TGTGACGATAGGACACTAGTCCTATCGTTTAATTTTTTAGCGACAAATTTGAATAAAATTACACACAGTAAAATCAAAGATTCCTAAACATAAAATATCTTAATGTGAAGGTGTGTTTTA TTGGCACACTAACTATAGCAATTATGTTCTTTGATTATAGAAAAAAAAAAAAAAAAAAAAA ATGGACCACTTCCACGAAATGATGGCCAGGACAGGTTCACATCCGACCAAAGCTGACATTCAGGAGTTCGTGAACCAGAACCTTGACCCCGAAGGATCTGAGTTCGAGGACTGGAGACCT
B

121

121

241

481

601

721

841

961

1081

1201

1321

1441

1561

1681

1801

1921

2041

2161

GTGTTGTGCCATTGTCGATAGTTTATTTATCGATAAGTGAAGATGTATAAGAAAATGTGGTGTGTTTTTGTTGCGATTATTGGAGTGGGTGGGGGGACGGACAGGAGCCATTTGCCGCCA ACCTGCTCTAGCAATATCTACTGTCATGGCCCGCTGCTGGACACGGGTGCAGATGGCAGGGCTGTACAATGACTCCAAGACCTTCGTTGACATGAAGCTGGAGCTGTCTGCCGACATCACC $T C S S N I Y C C H G F$ L D T Y Q M A G L Y T D S K T F V D M K L E L S A D I T M D H F H E M M A R T G S H P T K A D I G E F V N G N L D P E G S E F E D W R P ACTGACTGGAAGGATAACCCCGCGTTCCTACAAAACATCAAGGATCCATTGCTGCACGAATGGGCGGCCGACCTGAACCGCTTGTGGCTGCAGTTGGGCAGGAAGATGAAGCCTGATGTT

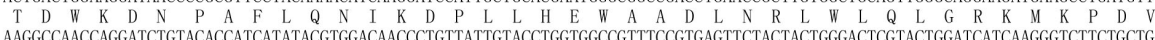
AAGGCCAACCAGGAACTGTACACCATCATATACGTGGACAACCCTGTTATTGTACCTGGTGGCCGTTTCCGTGAGTTCTACTACTGGGACTCGTACTGGATCATCAAGGGTCTTCTGCTC

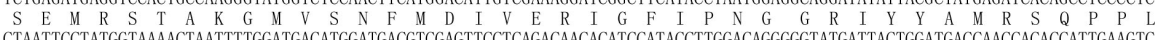
CTAATTCCTATGGTAAAACTAATTTTGGATGACATGGATGACGTCGAGTTCCTCAGACAACACATCCATACCTTGGACAGGGGGTATGATTACTGGATGACCAACCACACCATTGAAGTC

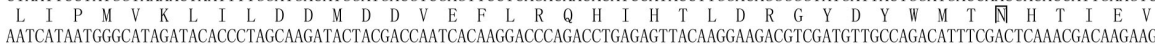
AATCATAATGGGCATAGATACACCCTAGCAGGATACTACGACCAATCACAAGGACCCAGACCTGAGAGTTACAAGGAGGACGTCGATGTTGCCAGACATTTCGACTCAAACGACAAGAAG GAAGAATTGTATGCCGAGCTGAAGGCTGCGGCTGAGTCCGGATGGGACTTCTCATCCAGATGGTTCATTCTTAATGGCACCAACAAAGGTAACCTAACGAACCTGAAAACTCGGTCCATC

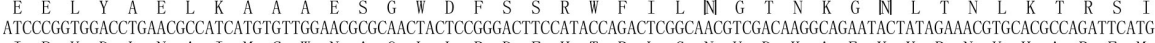
I P V D L N A I M C W N A Q L L R D F H T R L G N V D K A E Y Y R N V H A R F M

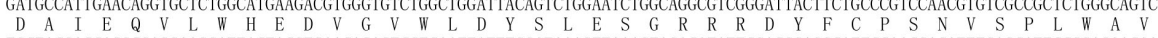
TGCTACGACCAGGCGAGGAAGGATTACTACGTGAACAGAGTCGTCAATTATTTGGATAAAGTTAAaGTAGACATATTCGACGGAGGCATTCCAACGACATTTGAGCATTCCGGAGAACAA

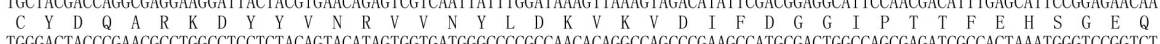
TGGGACTACCCGAACGCCTGGCCTCCTCTACAGTACATAGTGGTGATGGGCCCCGCCAACACAGGCCAGCCCGAAGCCATGCGACTGGCCAGCGAGATCGCCACTAAATGGGTCCGGTCT

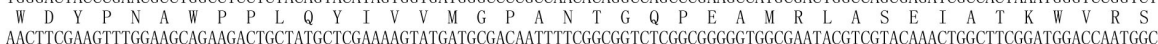
AACTTCGAAGTTTGGAAGCAGAAGACTGCTATGCTCGAAAAGTATGATGCGACAATTTTCGGCGGTCTCGGCGGGGGTGGCGAATACGTCGTACAAACTGGCTTCGGATGGACCAATGGC

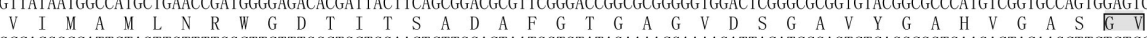
GCCACGGCCATTCTAGTTGTTTTGGCTTCTTTGGCTGCTGGAACTCTTGGACTAATGGTGTATAGAAAACGAAAAGATTACATCCGAGTCTCAGGCGGTGAAGACTACAAGCTTCTCTCC

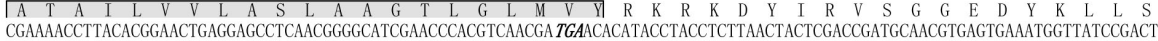

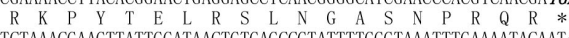

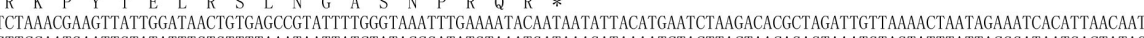
CTTCCAATGAATTGTATATTTGTGTTTTAAATAATTATCTATACCGATATGTAAATGATAAAGATAAAATCTACTTACTAACAGAGTAAATGTAGTATTTATTACGGATAATGACTATAC
ATGTCTAGGGTGTCACATTGGGGAAAAAAAAAAAAAAAAAA

Figure 1. A. Amino acid residues (1-23) are underlined and the arrowheads represent the signal peptide and putative cleavage site, respectively. Potential N-glycosylation sites (amino acid residues 51, 66, 77, 213, 240, and 336) are boxed. The initiation and termination codons are indicated in bold italic font. The probe sequence is between the arrowheads. The conserved sequence is shaded. B. Amino acid residues (1-18) are underlined and the arrowheads represent the signal peptide and putative cleavage site, respectively. Potential N-glycosylation sites (amino acid residues 49, 261, 331, and 337) are boxed. The initiation and termination codons are indicated in bold italic font. The probe sequence is between the arrowheads. The putative transmembrane region (residues 586-608) is shaded and boxed. The conservative sequence is shaded. 


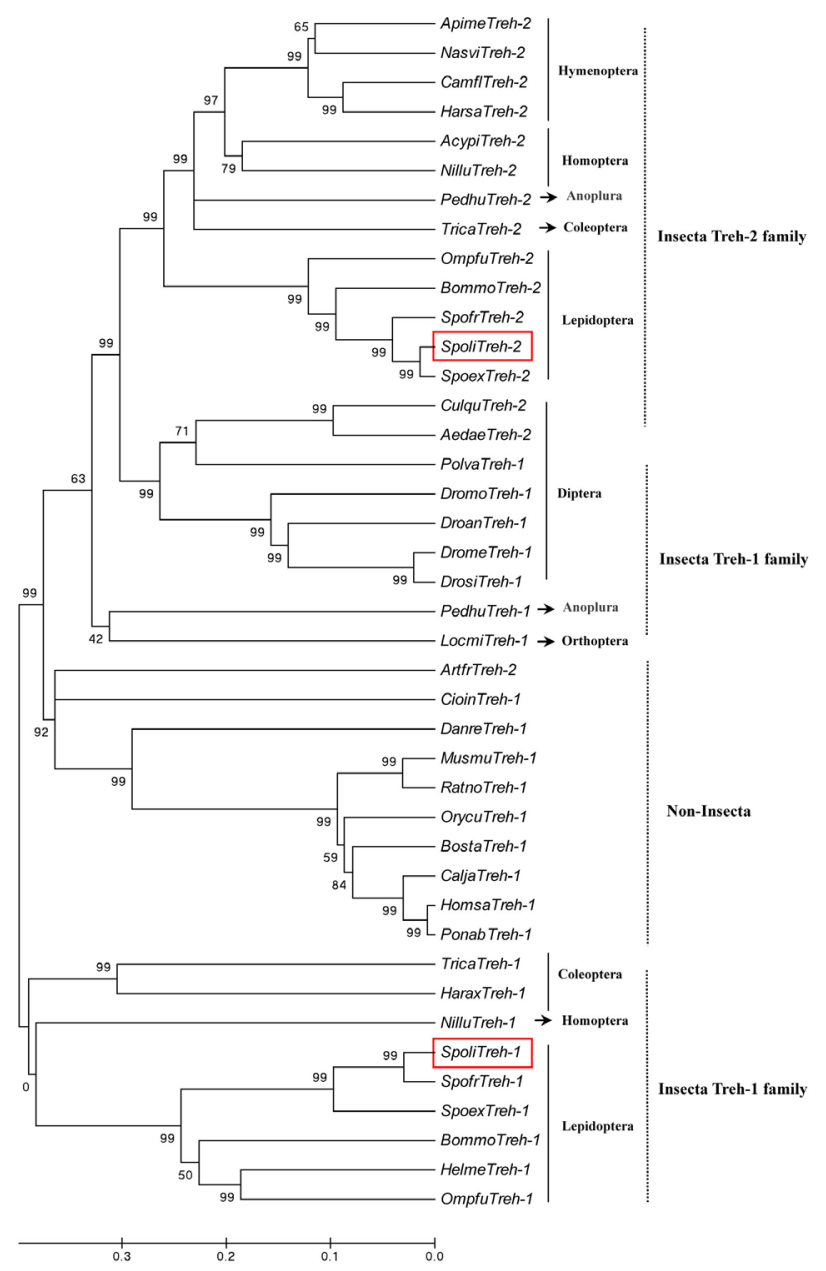

Figure 2. A phylogenetic tree was constructed based on the amino acid sequences of known Treh proteins. Fulllength amino acid sequences were aligned using the Mega 3.1 program. A bootstrap analysis was carried out and the robustness of each cluster was verified using 1000 replicates. Values at the cluster branches indicate the results of the bootstrap analysis. Treh proteins were from Acyrthosiphon pisum (Acypi, XM_001949424), Aedes aegypti (Aedae, XM_001660244), Apis mellifera (Apime, NM_001112671), Artemia franciscana (Artfr, AB059268), Bombyx mori (Bommo, NM_001043993 and NM_001043445), Bos taurus (Bosta, NM_001192570), Callithrix jacchus (Calja, XM 002754462), Camponotus floridanus (Camfl, GL434492), Ciona intestinalis (Cioin, XM_002131746), Culex quinquefasciatus (Culqu, XM_001847882), Danio rerio (Danre, XM_001336151), Drosophila ananassae (Droan, XM_001959628), D. melanogaster (Drome, NM_166423 and DQ864058), D. mojavensis (Dromo, XM_002005742), D. simulans (Drosi, DQ864075), Harmonia axyridis (Harax, HM056038), Harpegnathos saltator (Harsa, GL448204), Heliconius melpomene (Helme, HM056038), Homo sapiens (Homsa, NM_007180), Locusta migratoria (Locmi, FJ795020), Mus musculus (Musmu, NM_021481), Nasonia vitripennis (Nasvi, XM_001602129), Nilaparvata lugens (Nillu, FJ790319 and GQ397451), Omphisa fuscidentalis (Ompfu, EF426724 and EF426723), Oryctolagus cuniculus (Orycu, NM 001082290), Pediculus humanus (Pedhu, XM_002426623 and XP_002433202), Polypedilum vanderplanki (Polva, AB490335), Pongo abelii (Ponab, XM_002822558), Rattus norvegicus (Ratno, NM_001136141), Spodoptera exigua (Spoex, EU427311 and EU106080), Spodoptera frugiperda (Spofr, DQ447188 and EU872435), Spodoptera litura (Spoli, GU211890 and GU211891), and Tribolium castaneum (Trica, XM_968826 and XM_967517). 
to S. frugiperda Treh1 (see Table 2). SpoliTreh2 and S. exigua Treh2 shared $96 \%$ sequence homology. All Treh2 genes had 1 or 2 transmembrane domains, including some species with a potential transmembrane helical domain in Treh2 (see Table 3). The deduced amino acid sequence of SpoliTreh1 was aligned with Treh1 and Treh2 genes from other species (Figure 3).

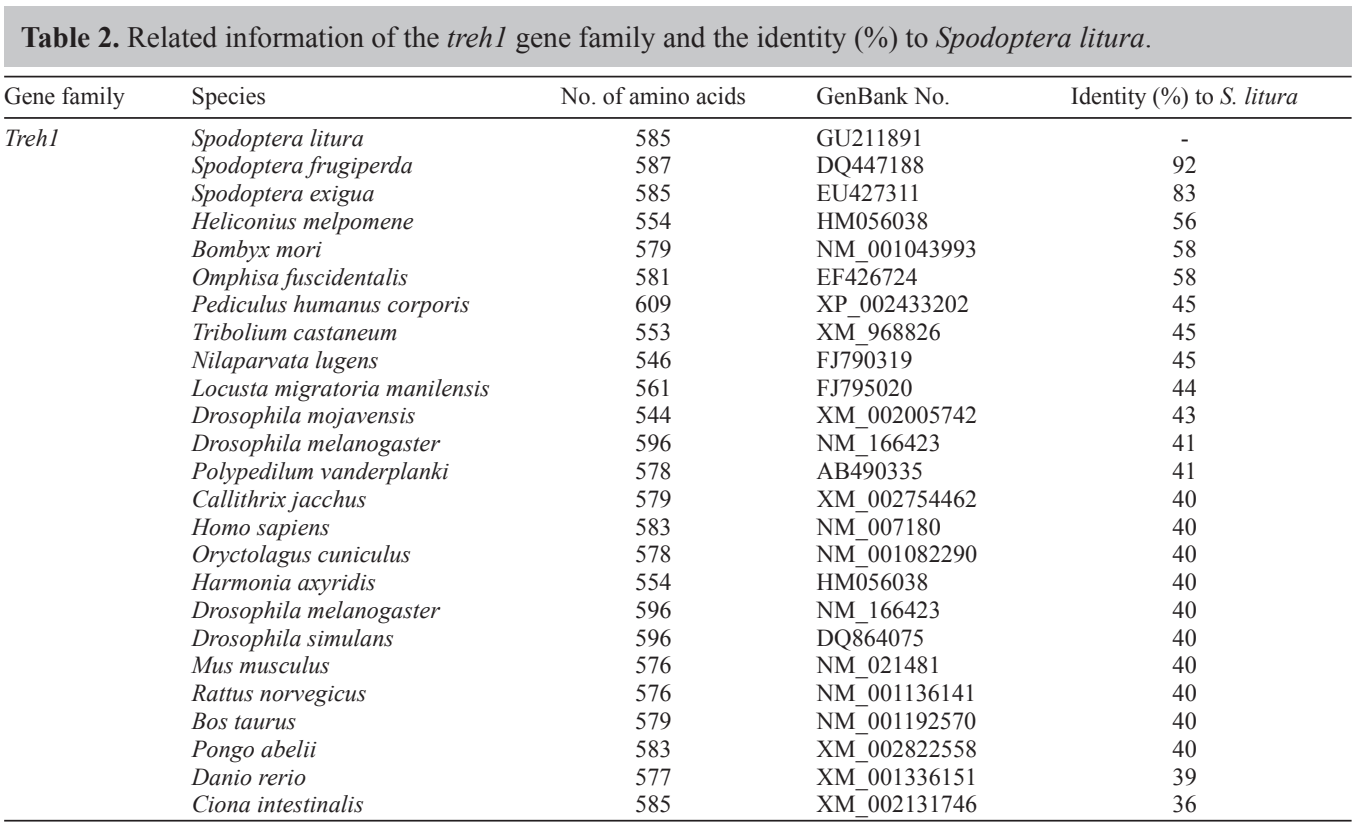

Table 3. Related information of the treh2 gene family and the identity (\%) to Spodoptera litura.

\begin{tabular}{llllll}
\hline Gene family & Species & $\begin{array}{c}\text { No. of amino } \\
\text { acids }\end{array}$ & GenBank No. & $\begin{array}{c}\text { Identity (\%) to } \\
\text { S. litura }\end{array}$ & Transmembrane domain analysis \\
\hline Treh2 & S. litura & 645 & GU211890 & - & $585-607$ \\
& S. exigua & 645 & EU106080 & 96 & $585-607$ \\
S. frugiperda & 647 & EU872435 & 91 & $587-609$ \\
Bombyx mori & 642 & NM_001043445 & 76 & $582-604$ \\
Omphisa fuscidentalis & 648 & EF426723 & 74 & $581-603$ \\
Apis mellifera & 626 & NM_001112671 & 53 & $13-32$ and 594-616 \\
Tribolium castaneum & 632 & XM_967517 & 52 & $575-597$ \\
Nilaparvata lugens & 665 & GQ397451 & 51 & $598-620$ \\
Acyrthosiphon pisum & 625 & XM_001949424 & 51 & Potential transmembrane helical domain \\
Camponotus floridanus & 665 & GL434492 & 50 & $13-32$ and 599-621 \\
Harpegnathos saltator & 668 & GL448204 & 50 & $12-34$ and 606-625 \\
Pediculus humanus corporis & 650 & XM_002426623 & 49 & Potential transmembrane helical domain \\
Nasonia vitripennis & 671 & XM_001602129 & 48 & $600-622$ \\
Culex quinquefasciatus & 586 & XM_001847882 & 46 & Potential transmembrane helical domain \\
Aedes aegypti & 618 & XM_001660244 & 46 & $12-34$ and 590-612 \\
Artemia franciscana & 703 & AB059268 & 41 & Potential transmembrane helical domain \\
\hline
\end{tabular}

\section{Structure of SpoliTreh proteins}

We chose 5 species of animals to ensure the reliability of the alignment. Multiple 
sequence alignment of Treh proteins revealed a high degree of conservation (Figure 3). Alignment of proteins from insects revealed that Treh proteins have many conserved motifs, including KTFVD, PGGRFR (I)EFYYWDS(T)YWI, PRPESY, AESGWDFSSRW, MF(Y)EKY, and GGEYEVQTGFGWS(T)NG (see Figure 3). SpoliTreh1 has 6 potential N-glycosylation sites (residues 51, 66, 77, 213, 240, and 336) and SpoliTreh2 has 4 sites (residues 49, 261, 331, and 337). The first 23 amino acids were identified as the signal peptide, in which SpoliTreh2 displayed a signal peptide of 18 amino acids. In addition, the alignment of SpoliTreh2 with other insect trehalases showed that it contained 2 signature motifs in the trehalase and a putative transmembrane domain (residue 586-608), which is an important characteristic of Treh2 (see Table 2).

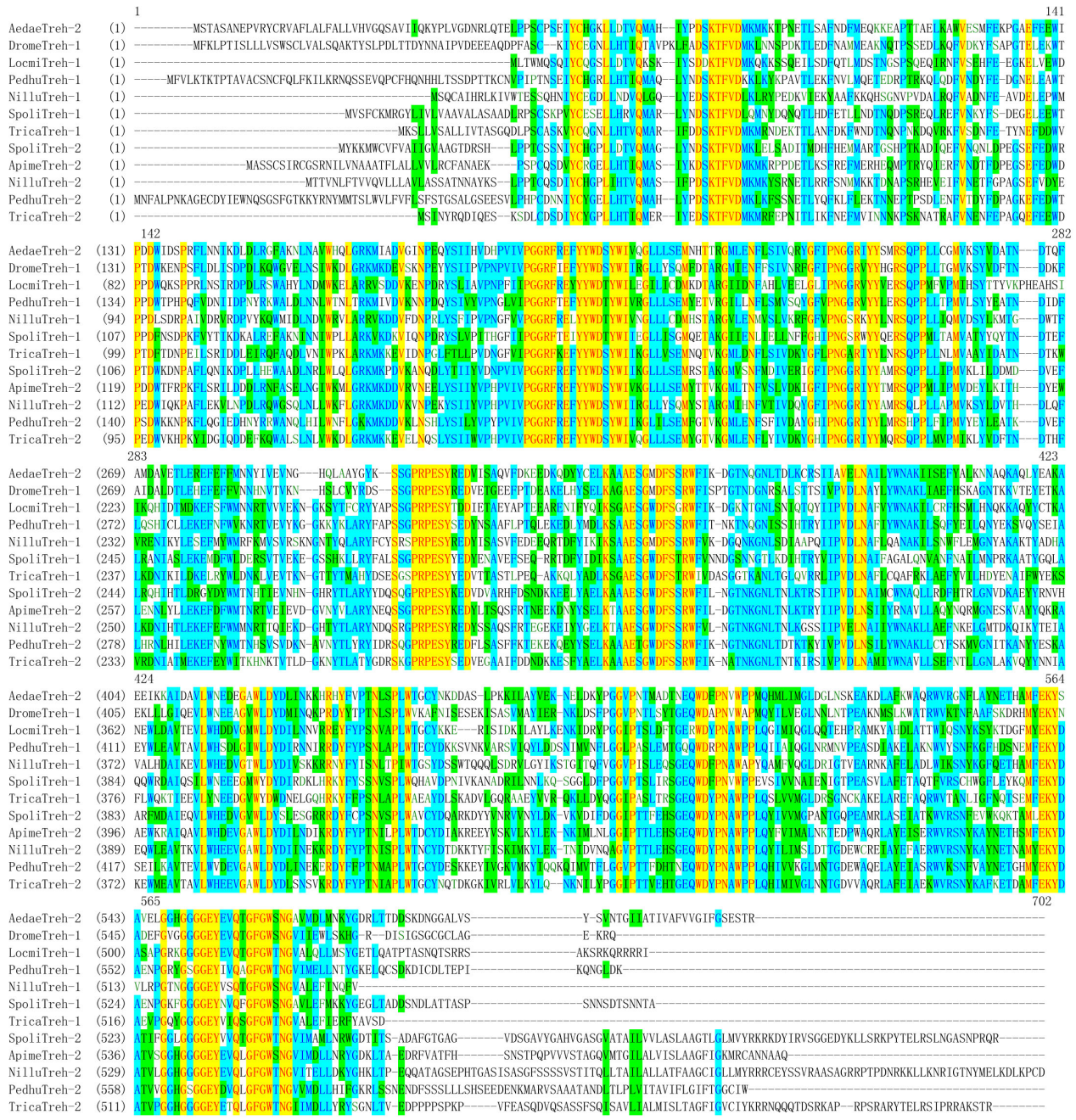

Figure 3. Alignment of DromeTreh-1, LocmiTreh-1, NilluTreh-1, PedhuTreh-1, SpoliTreh-1, TricaTreh-1, AedaeTreh-2, ApimeTreh-2, NilluTreh-2, PedhuTreh-2, SpoliTreh-2, and TricaTreh-2 proteins using the Vector NTI 9.0 software. Highly conserved regions are shown in yellow and sky-blue. 


\section{Treh tissue distribution in S. litura}

SpoliTreh tissue-specific expression was determined with Northern blotting. SpoliTreh1 transcripts were detected in midgut, fat body, trachea, and epidermis. The highest expression of SpoliTreh1 occurred in the midgut and decreased rapidly in nearly all other tissues, whereas it was absent in the brain and Malpighian tubules of S. litura larvae. SpoliTreh 2 transcripts were detected in all 6 of the sampled tissues, including the fat body, midgut, Malpighian tubules, tracheae, brain, and epidermis of S. litura larvae. The expression in the midgut, Malpighian tubules, and epidermis was higher than that in other tissues but lower than SpoliTreh1 expression in the midgut (Figure 4).

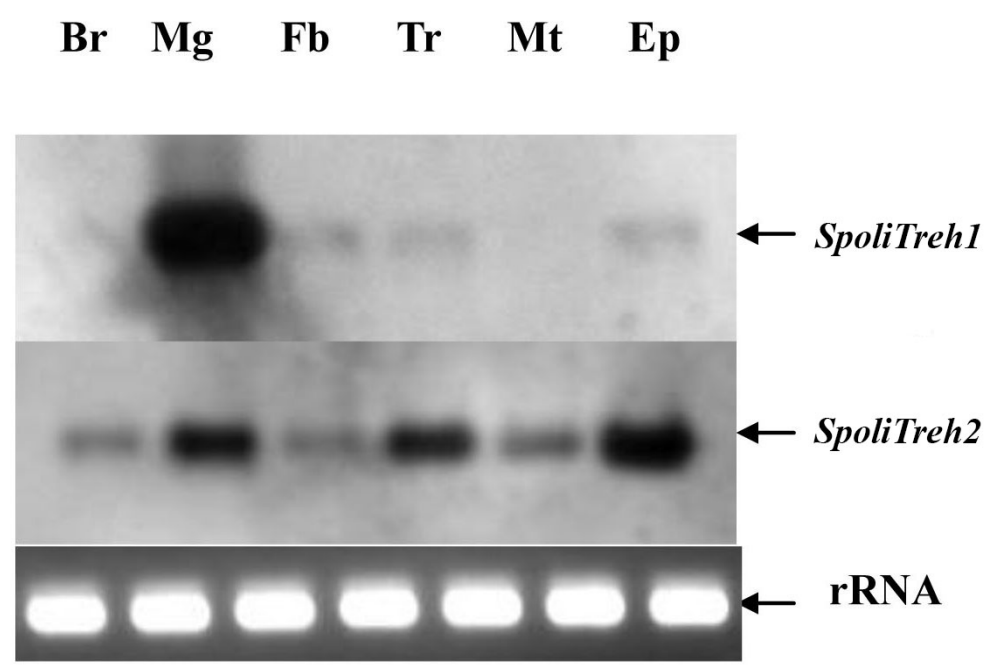

Figure 4. Northern blot analysis of Spolitreh transcripts in different tissues from 3-day-old larvae of 5th instar. Total RNA was extracted from various tissues: brain $(\mathrm{Br})$, midgut $(\mathrm{Mg})$, fat body $(\mathrm{Fb})$, tracheae $(\mathrm{Tr})$, Malpighian tubules $(\mathrm{Mt})$, and epidermis (Ep). Treh-specific probes were marked with digoxin. Following hybridization and detection using colored substrate solution, the membrane was stripped by boiling in $0.1 \%$ SDS. rRNA was used as a control.

\section{Developmental SpoliTreh expression}

Northern blot experiments were carried out to analyze SpoliTreh expression patterns in the fat body from the 6th-instar larval stage to the pupal stage and in the whole body from the 1st- to 5th-instar larval stage of $S$. litura. SpoliTreh mRNAs were expressed at different levels in the fat body and the whole body. Compared to expression in other stages, SpoliTreh1 transcripts were expressed more highly in the fat body in the pre-pupal stage, and SpoliTreh2 transcripts were highly expressed in the fat body on the 3-day-old 6th-instar larval stage and in 1-, 3-, 4-, 5-, and 6-day-old pupae (Figure 5A).

Figure 5B shows the expression in the larval stages from the 1st to the 5th instar. Expression results of the whole body revealed that SpoliTreh1 and SpoliTreh2 mRNA was highly expressed in the 3rd-instar larval stage. SpoliTreh1 mRNA was highly expressed on day one of the 2nd-instar larval stage. SpoliTreh 2 was highly expressed on day 2 of the 1st- and 2nd-instar larval stage and on days 1 and 2 of the 4th-instar larval stage (Figure 5B). 
A
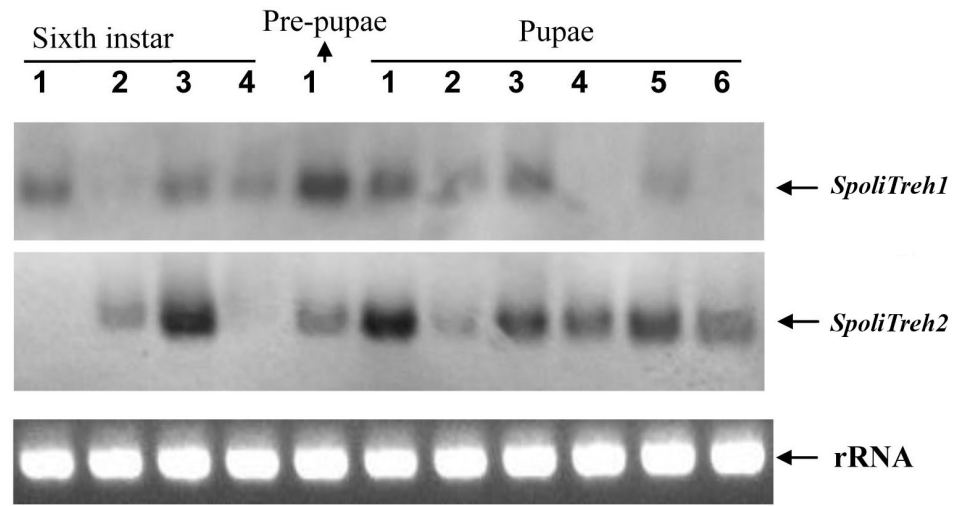

B

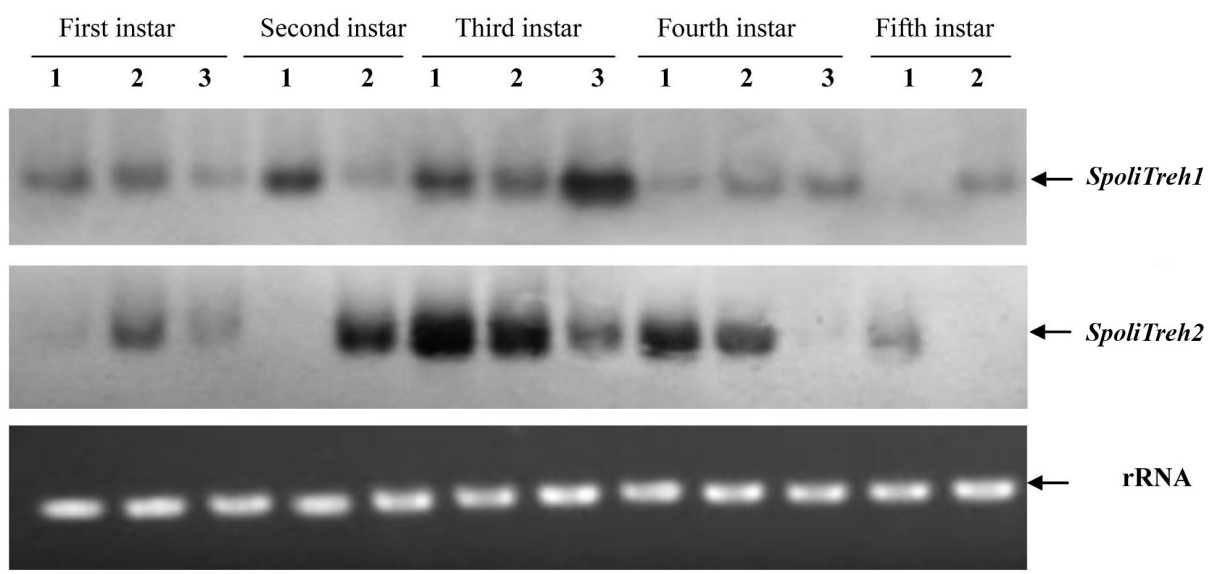

Figure 5. A. Developmental expression of Spolitreh1 and Spolitreh2 in the fat bodies of 6th-instar larvae, pre-pupae and pupae. rRNA was used as a control. Numbers 1-6 denote days 1-6 of the developmental stage, respectively. B. Developmental expression of Spolitreh1 and Spolitreh2 in the whole body of 1st-, 2nd-, 3rd-, 4th-, and 5th-instar larvae. rRNA was used as a control. Numbers 1-3 denote days 1-3 of the developmental stage, respectively.

\section{DISCUSSION}

The first insect trehalase gene was cloned in T. molitor, and B. mori trehalase genes were cloned soon thereafter (Takiguchi et al., 1992; Su et al., 1993). In this study, we cloned the cDNA sequences of Treh1 and Treh2 genes in S. litura, and the protein sequence translation analysis indicated that Treh1 and Treh2 coded for soluble and membrane-bound trehalase, respectively (GenBank accession Nos. GU211891 and GU211890). SpoliTreh1 cDNA has a predicted mass of approximately $67.07 \mathrm{kDa}$ (Figure 1A), which is similar to the molecular weights of the soluble trehalase purified from the pupal midgut of B. mori $(67-70 \mathrm{kDa})$ and the larval midgut of $O$. fuscidentalis (65.2 kDa) (Sumida and Yamashita, 1983; Tatun et al., 2008). The molecular mass of SpoliTreh2 cDNA is similar to the molecular mass of Bmtreh-2 (71 and $74 \mathrm{kDa}$ ). Our results are almost identical to those of Tang et al. (2008). 
In this study, comparison and analysis of Treh homology based on the DNA and proteins of several orders of insects found that SpoliTreh genes shared close homology with Lepidoptera, and the same types of Treh showed higher homology than that between Treh1 and Treh2 (Figure 2). Results from software analysis of each gene include a figure and corresponding description, the figures from software analysis of Acyrthosiphon pisum Treh2, Artemia franciscana Treh2, Culex quinquefasciatus Treh2, and Pediculus humanus corporis Treh2 revealed that they have 1 or 2 potential transmembrane helical domains, indicated by a red crest (O. fuscidentalis has no potential transmembrane helical domains as a contrast in order to see clearly); however, their corresponding description from software analysis do not show what they do (Figure S1). The potential transmembrane helical domain may be related to species evolution or have unknown functions that deserve further investigation.

Trehalases in all insects have some common features: they contain a leading signal peptide area, a field coil, a highly conserved glycine-rich region, and 2 conserved signal sequences. SpoliTreh2 displayed 4 potential N-glycosylation sites, whereas SpoliTreh1 had more. In addition, the alignment of SpoliTreh2 with other insect trehalases showed that it contained 2 signature motifs and a putative transmembrane helical domain (residues 585-607) that was absent in SpoliTreh1 but was an important characteristic of Treh2. This result was consistent with a study of trehalase genes cloned in S. exigua (Tang et al., 2008). The comparison of 2 trehalase mRNAs and proteins revealed their respective characteristics, which may indicate differences in function between the 2 trehalases and lay the foundation for further study of trehalose metabolism regulation.

Almost all of the genes encoding the soluble trehalase were mainly expressed in the epidermis tissue, midgut, Malpighian tubules, and ovary during the larval stage ( $\mathrm{Su}$ et al., 1993, 1994; Kamimura et al., 1999; Parkinson et al., 2003). Other studies have determined that Treh2 and Treh1 genes in T. molitor and B. mori were completely different (Su et al., 1993, 1994; Sato et al., 1997). In this study, we adopted Northern blotting hybridization combined with reverse transcription PCR to determine tissue distribution. Results of these analyses showed that fifth-instar larval S. litura Treh1 was expressed in the midgut, fat body, tracheae, and epidermis but not in the brain and Malpighian tubules. S. litura Treh2 expressed in each of these tissues, whereas previous research results have shown that in S. exigua, Treh2 distribution was confined to the midgut, fat body, and Malpighian tubules and was not detected in the tracheae, epidermis, and brain (Tang et al., 2008). This finding indicated that Treh2 in 2 species differed in expression and tissue distribution even though the species belong to the same order. The results also indicated that the 2 trehalases may serve different functions in these 6 tissues during the development of these Lepidopteran insects.

We do not know whether the expression pattern and function of the 2 trehalases are different or play several functions in all Lepidopteran insects. Treh1 expression in the midgut was higher compared to that in other tissues, which was consistent with other studies and indicated its characteristic function (Mitsumasu et al., 2005; Tatun et al., 2008). The consistent but different levels of Treh 2 expression in the 6 tissues indicate that it plays an irreplaceable role in hydrolyzing the trehalose consumed by larvae from food because Treh2 is an exogenous enzyme. Treh1 and Treh2 were distributed differently in tissue, which indicated that they have tissue-specific expression and play distinct roles.

We wanted to examine the dynamic expression of trehalases from the larval to the pupal stages, but many organs of the pupae degenerated; in fact, S. litura pupae had many fat bodies, so only fat bodies from the 6th-larval stage to the pupal stage were selected. In this study, SpoliTreh1 
and Treh 2 in the fat body of the pre-pupal stage were highly expressed. This dramatic increase in expression is needed to prepare sufficient energy for pupation and hydrolyze trehalose for chitosan biosynthesis. These findings agreed with those of Chen et al. (2010) that Trehl regulates the chitosan biosynthesis in the epidermis. The reduction of Treh1 was related to the fact that storage was sufficient and the insect required less energy in pupal stage (Figure 5A). Treh2 in the fat body was expressed more highly in the pupal stage, which may promote chitosan in gut consistent with the findings of Chen et al. (2010) that Treh2 regulates chitosan biosynthesis in the gut. Treh1 and Treh 2 were both highly expressed in 3rd-instar larvae. Treh1 on day 1 of the 2nd-larval stage showed high expression, as did Treh2 on day 2 of the 2nd-larval stage and on days 1 and 2 of the 4th larval stage (Figure 5A). The expression of the 2 trehalases was similar to that in $S$. exigua (Chen et al., 2010). These results also demonstrated that SpoliTreh1 and SpoliTreh2 had different functions at different developmental stages. Treh1 and Treh2 hydrolyzed trehalose into glucose to provide the larvae with energy for growth, but they may have other roles. Our study found that Treh1 influenced the expression of chitin synthase A, which is responsible for chitin synthesis in the cuticle and tracheae, whereas Treh2 influenced the expression of chitin synthase B, which is responsible for chitin synthesis in the midgut in S. exigua (Chen et al., 2010). The larvae synthesized a high level of chitin synthase A for several ecdyses during the larval stages, so the expression of Trehl was constant except for some fluctuations and decreased in pupae when S. litura did not undergo ecdyses. Treh 2 contributed to the development of the midgut. Treh1 and Treh2 also hydrolyzed trehalose into energy for the insect growth. Meanwhile, the dynamic expression level of 2 trehalases was related to the regulation of trehalose and glucose.

Accordingly, we concluded that Treh1 and Treh2 had their own characteristic as well as some similar functions, regulating trehalose concentration together in some tissues and individually in others. They regulate trehalose together in the life of insect such that Treh1 mainly hydrolyzes trehalose intracellularly, and Treh2 acts on intracellular and extracellular trehalose. However, the precise function of these genes remains unknown and worth further research. For its important role in S. litura, trehalases that can block trehalase enzyme synthesis when its expression is high enough may provide a new target for pest control without environmental pollution.

\section{ACKNOWLEDGMENTS}

Research supported by the National Natural Science Foundation of China (Grants \#31000880 and \#30970473), the Qianjiang Talents Project of the Technology Office in Zhejiang Province (\#2010R10093), the Zhejiang Provincial Natural Science Foundation of China (Grants \#Y3100176 and \#Y307551), the Middle-Aged and Young Talents Subject Leaders in Zhejiang Province, The Project of Zhejiang Key Scientific and Technological Innovation Team (Grant \#2010R50039), the Hangzhou Normal University High-Level Talents Start-Up Fund (Grant \#YS05203105), and the Program for Excellent Young Teachers in Hangzhou Normal University (Grant \#JTAS 2011-01-031).

\section{Supplementary material}

\section{REFERENCES}

Chen J, Tang B, Chen H, Yao Q, et al. (2010). Different functions of the insect soluble and membrane-bound trehalase genes in chitin biosynthesis revealed by RNA interference. PLoS One 5: e10133. 
Crowe JH, Crowe LM and Chapman D (1984). Preservation of membranes in anhydrobiotic organisms: the role of trehalose. Science 223: 701-703.

Davidson P and Sun WQ (2001). Effect of sucrose/raffinose mass ratios on the stability of co-lyophilized protein during storage above the Tg. Pharm. Res. 18: 474-479.

de Almeida FM, Bonini BM, Beton D, Jorge JA, et al. (2009). Heterologous expression in Escherichia coli of Neurospora crassa neutral trehalase as an active enzyme. Protein Expr. Purif. 65: 185-189.

Elbein AD, Pan YT, Pastuszak I and Carroll D (2003). New insights on trehalose: a multifunctional molecule. Glycobiology 13: 17R-27R.

Eleutherio EC, Araujo PS and Panek AD (1993). Role of the trehalose carrier in dehydration resistance of Saccharomyces cerevisiae. Biochim. Biophys. Acta 1156: 263-266.

Frison M, Parrou JL, Guillaumot D, Masquelier D, et al. (2007). The Arabidopsis thaliana trehalase is a plasma membranebound enzyme with extracellular activity. FEBS Lett. 581: 4010-4016.

Kamimura M, Takahashi M, Tomita S, Fujiwara H, et al. (1999). Expression of ecdysone receptor isoforms and trehalase in the anterior silk gland of Bombyx mori during an extra larval molt and precocious pupation induced by 20-hydroxyecdysone administration. Arch. Insect Biochem. Physiol. 41: 79-88.

Lee JH, Tsuji M, Nakamura M, Nishimoto M, et al. (2001). Purification and identification of the essential ionizable groups of honeybee, Apis mellifera L., trehalase. Biosci. Biotechnol. Biochem. 65: 2657-2665.

Lee JH, Saito S, Mori H, Nishimoto M, et al. (2007). Molecular cloning of cDNA for trehalase from the European honeybee, Apis mellifera L., and its heterologous expression in Pichia pastoris. Biosci. Biotechnol. Biochem. 71: 2256-2265.

Mariano AC, Santos R, Gonzalez MS, Feder D, et al. (2009). Synthesis and mobilization of glycogen and trehalose in adult male Rhodnius prolixus. Arch. Insect Biochem. Physiol. 72: 1-15.

Mitsumasu K, Azuma M, Niimi T, Yamashita O, et al. (2005). Membrane-penetrating trehalase from silkworm Bombyx mori. Molecular cloning and localization in larval midgut. Insect Mol. Biol. 14: 501-508.

Mitsumasu K, Azuma M, Niimi T, Yamashita O, et al. (2008). Changes in the expression of soluble and integral-membrane trehalases in the midgut during metamorphosis in Bombyx mori. Zoolog. Sci. 25: 693-698.

Parkinson NM, Conyers CM, Keen JN, MacNicoll AD, et al. (2003). cDNAs encoding large venom proteins from the parasitoid wasp Pimpla hypochondriaca identified by random sequence analysis. Comp. Biochem. Physiol. C. Toxicol. Pharmacol. 134: 513-520.

Sato K, Komoto M, Sato T, Enei H, et al. (1997). Baculovirus-mediated expression of a gene for trehalase of the Mealworm Beetle, Tenebrio molitor, in insect cells, SF-9, and larvae of the cabbage armyworm, Mamestra brassicae. Insect Biochem. Mol. Biol. 27: 1007-1016.

Silva MC, Terra WR and Ferreira C (2010). The catalytic and other residues essential for the activity of the midgut trehalase from Spodoptera frugiperda. Insect Biochem. Mol. Biol. 40: 733-741.

$\mathrm{Su}$ ZH, Sato Y and Yamashita O (1993). Purification, cDNA cloning and northern blot analysis of trehalase of pupal midgut of the silkworm, Bombyx mori. Biochim. Biophys. Acta 1173: 217-224.

$\mathrm{Su} \mathrm{ZH}$, Ikeda M, Sato Y, Saito H, et al. (1994). Molecular characterization of ovary trehalase of the silkworm, Bombyx mori and its transcriptional activation by diapause hormone. Biochim. Biophys. Acta 1218: 366-374.

Sumida M and Yamashita O (1977). Trehalase transformation in silkworm midgut during metamorphosis. J. Comp. Physiol. 115: 241-253.

Sumida M and Yamashita O (1983). Purification and some properties of soluble trehalase from midgut of pharate adult of the silkworm, Bombyx mori. Insect Biochem. 13: 257-265.

Sun WQ and Davidson P (1998). Protein inactivation in amorphous sucrose and trehalose matrices: effects of phase separation and crystallization. Biochim. Biophys. Acta 1425: 235-244.

Takiguchi M, Niimi T, Su ZH and Yaginuma T (1992). Trehalase from male accessory gland of an insect, Tenebrio molitor. cDNA sequencing and developmental profile of the gene expression. Biochem. J. 288: 19-22.

Tang B, Chen X, Liu Y, Tian H, et al. (2008). Characterization and expression patterns of a membrane-bound trehalase from Spodoptera exigua. BMC Mol. Biol. 9: 51.

Tatun N, Singtripop T, Tungjitwitayakul J and Sakurai S (2008). Regulation of soluble and membrane-bound trehalase activity and expression of the enzyme in the larval midgut of the bamboo borer Omphisa fuscidentalis. Insect Biochem. Mol. Biol. 38: 788-795.

Ujita M, Yamanaka M, Maeno Y, Yoshida K, et al. (2011). Expression of active and inactive recombinant soluble trehalase using baculovirus-silkworm expression system and their glycan structures. J. Biosci. Bioeng. 111: 22-25.

Wegener G, Tschiedel V, Schloder P and Ando O (2003). The toxic and lethal effects of the trehalase inhibitor trehazolin in locusts are caused by hypoglycaemia. J. Exp. Biol. 206: 1233-1240.

Wyatt GR (1967). The biochemistry of sugars and polysaccharides in insects. Adv. Insect Physiol. 4: 287-360. 
Yamashita O, Sumida M and Hasegawa K (1974). Developmental changes in midgut trehalase activity and its localization in the silkworm, Bombyx mori. J. Insect Physiol. 20: 1079-1085.

Yamoah E, Jones EE, Weld RJ, Suckling DM, et al. (2008). Microbial population and diversity on the exoskeletons of 4 insect species associated with gorse (Ulex europaeus L.). Aust. J. Entomol. 47: 370-379. 